\title{
Primary syphilis in the oral cavity
}

\author{
NICHOLAS J. FIUMARA A AND MONICA BERG† \\ Department of Dermatology and Syphilology, Boston Dispensary, Tufts New England Medical Center, U.S.A.
}

The apparent increase in oro-genital activity in both homosexual and heterosexual population groups has focused the physician's attention on a neglected part of the anatomy. As a result, gonococcal pharyngitis was rediscovered (Fiumara, 1971). With about three million people infected with gonorrhoea each year in the United States, it is understandable that there is no dearth of patients recognized as having gonococcal pharyngitis. On the other hand, with only about 80,000 cases of primary and secondary syphilis occurring each year, the chance of primary syphilis occuring in the mouth would be infrequent, so that these lesions sometimes go unrecognized.

The diagnosis of primary syphilis in the mouth of a patient seen recently has prompted this report of three patients with primary syphilis of the oral cavity which were recognized by us in 1973 .

\section{Case reports}

Case 1, a 22-year-old male homosexual, came to our clinic on December 7, 1973, because his room-mate had gonococcal urethritis. The patient complained of a sore throat of 1 week's duration. On examination of the mouth, an erosive lesion was discovered on the hard palate opposite the right upper second molar (Fig. 1). The lesion was not tender. Diffuse oedema, erythema, and punctate pustules were seen in the tonsillar area. A culture from the tonsillar area was taken and blood for a RPR card test. $N$. gonorrhoeae was grown and the patient was given $4 \mathrm{~g}$. spectinomycin intramuscularly.

On December 11, 1973, the RPR card test was reported reactive at $1: 4$ and the FTA-ABS test was $2+$ reactive. The patient was immediately sent for and he returned to the clinic on December 18, 1973.

On examination, the oedema and erythema of the oropharynx had completely subsided, but the erosive lesion on the hard palate was unchanged. He complained of feeling a 'lump' on the roof of the mouth, but it did not hurt him. The erosion was non-tender on examination with a gloved finger; the anterior cervical glands were

Received for publication February 1, 1974

$\star$ Director, Division of Communicable Diseases, Massachusetts Department of Public Health.

†Dermatologist, Boston Dispensary, Tufts New England Medical Center.

Address: Prof. N. J. Fiumara, Department of Public Health, 600

Washington Street, Boston 02111, U.S.A.

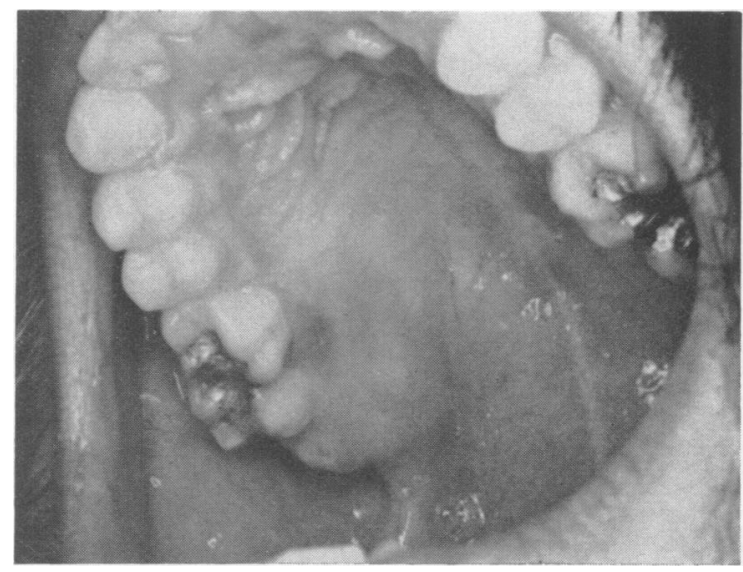

FIG. 1 Case 1. Primary lesion on hard palate opposite right upper second molar

enlarged and non-tender. A careful examination of the entire body revealed no evidence of a rash or other adenopathy. A darkfield examination of the lesion was not made. Primary syphilis was diagnosed, and the patient was given 2.4 m.u. benzathine pencillin $G$ intramuscularly which was repeated 1 week later. A pre-treatment RPR card test taken on December 18, 1973, was reactive in a dilution of $1: 128$ and the FTA-ABS test was now $4+$ reactive. When the patient received his second injection on December 27,1973 , the erosive lesion on the hard palate had practically healed.

Case 2, a 24-year-old male homosexual, came to the clinic on March 1, 1973, with his friend who was under treatment for secondary syphilis. His only complaint was that he had a lump on the left side of his throat which did not hurt him, did not interfere with eating or drinking, and had been present for about a week or 10 days. Erythema and oedema were present in the left tonsillar area with the anterior pillar pushed forward (Fig. 2, overleaf).

There was also a hard non-tender ulcer with induration of the tonsillar pillar. The anterior cervical glands were enlarged, hard, and non-tender. The blood RPR card test was reactive in a dilution of $1: 64$ and the FTA-ABS test was $4+$ reactive. A diagnosis of primary syphilis was made. 


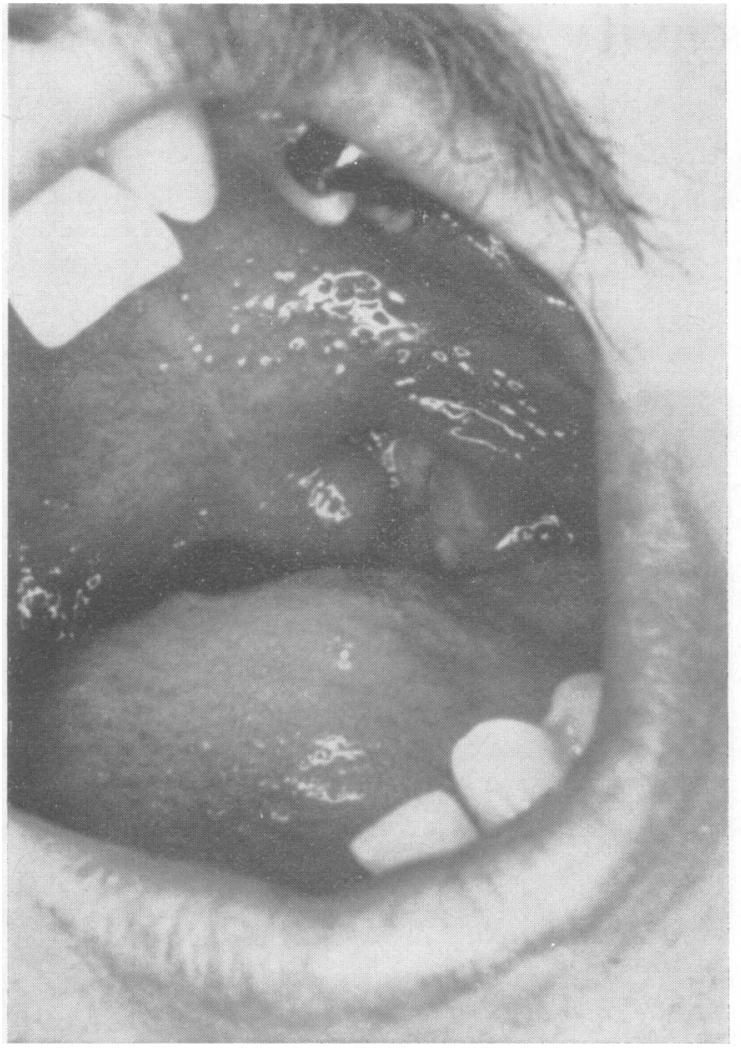

FIG. 2 Case 2. Primary lesion of the left tonsil

Case 3, a 28-year-old male homosexual, attended on July 8,1973 . His history mimicked that of Case 2 in that he was a contact of a patient with secondary syphilis and his only complaint was a lump in the left side of the throat (Fig. 3). The findings on examination were exactly the same as in Case 2.

\section{Discussion}

While most chancres appear on the genitals, they may be seen anywhere on the body, particularly in the erotic areas. The lips are the commonest site of chancres of the oral cavity (Fiumara, 1972), which may be the result of oral-genital sex or kissing. Most chancres in the male tend to be located on the upper lip; in the female on the lower lip. The anterior cervical glands in such cases are enlarged and nontender. Chancres of the tongue, which are next in frequency, take the form of painless indurated ulcers.

The tonsillar area, next in frequency to the lips and tongue, is another common location for oral chancres; there is considerable oedema, redness, and an

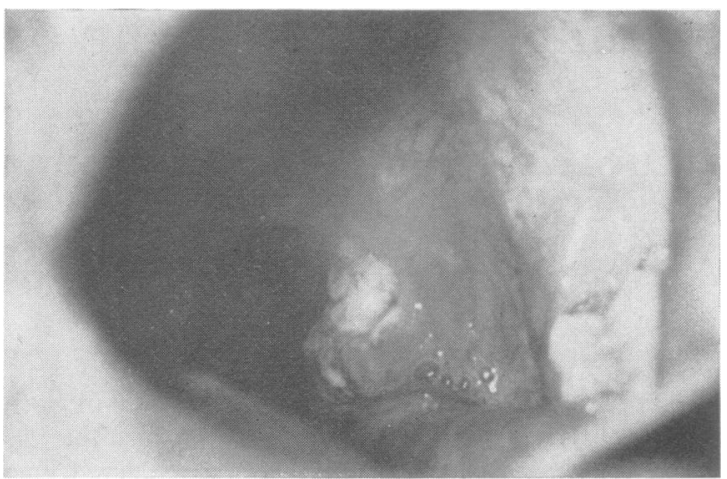

FIG. 3 Case 3. Primary lesion of the left tonsil

ulcerated or eroded lesion. The uvula may be pushed over to the uninvolved side; the anterior tonsillar pillar is red, swollen, and anteriorly displaced. The adjacent area of the tongue may be affected, with enlargement of the foliate papillae. The hallmark of syphilis is the absence of pain, and the patient complains of a persistent painless 'lump' in the throat. Every case of tonsillar syphilis that $I$ have seen in 34 years of practice involved the left tonsil, but I have no explanation of this observation.

The oral lesions of primary syphilis are teeming with spirochaetes, yet the only site where a darkfield examination may be performed with meaningful results is on the lips. One cannot distinguish with certainty in a darkfield examination $T$. microdentium, which normally exists in the mouth, from $T$. pallidum. Confirmation of the clinical diagnosis must therefore rest on the reagin and treponemal blood tests.

\section{Summary}

Three cases of primary syphilis of the oral cavity are described. With the apparent increase in oro-genital sexual practices, such cases are likely to be seen more frequently.

\section{References}

Fiumara, N. J. (1971) Medical Aspects of Human Sexuality, 5, no. 5, p. 195

- (1972) Ibid., 6, no. 9, p. 68

\section{Syphilis primaire de la cavité buccale}

On décrit trois cas de syphilis primaire de la cavité buccale. Avec l'apparente augmentation des pratiques sexuelles orogénitales, il est probable que de tels cas seront recontrés plus fréquemment. 